\title{
Teoría de corte-fricción aplicada al hormigón reforzado con fibras de acero
}

\author{
A. Picazo ${ }^{1}$, J.C. Gálvez ${ }^{2 *}$, M.G. Alberti ${ }^{2}$, A. Enfedaque ${ }^{2}$ \\ *Autor de Contacto: jaime.galvez@upm.es \\ ${ }^{1}$ Departamento de Tecnología de la Edificación. E.T.S. de Edificación. Universidad Politécnica de Madrid. Avda. \\ Juan de Herrera 6, 28040 Madrid-España. \\ ${ }^{2}$ Departamento de Ingeniería Civil: Construcción. E.T.S. de Ingenieros de Caminos, Canales y Puertos. Universidad \\ Politécnica de Madrid. C/ Profesor Aranguren, s/n, 28040 Madrid-España.
}

\section{RESUMEN}

El hormigón reforzado con fibras de acero (HRFA) puede tener carácter estructural si cumple los requisitos establecidos por las normas de hormigón estructural basadas en resistencias residuales en ensayos de fractura a flexión. Sin embargo, para fractura a cortante el uso de dichas resistencias sigue estando cuestionado. Por esto los mecanismos resistentes de engranamiento de áridos y efecto pasador de la armadura conforme a la teoría de corte-fricción en HRFA sigue siendo objeto de estudio. Cabe recordar que las fibras mejoran el comportamiento del material frente a tensiones de corte en una grieta ya que cosen los labios de la fisura, reduciendo su apertura y aumentando el rozamiento del material. Con estos antecedentes, se diseñó y desarrolló una campaña experimental sobre HRFA en probetas tipo "push-off" sometidas a esfuerzos de corte directo para la obtención de los desplazamientos relativos entre los lados de las fisuras y así estudiar la validez de la teoría de corte-fricción desarrollada por Walraven. Del estudio comparativo entre los resultados experimentales y el modelo analítico de corte-fricción se concluye que el modelo analítico propuesto es apto al aplicarlo al comportamiento del HRFA, una vez creada la fisura de cortante, reproduciendo de forma adecuada los desplazamientos normales y tangenciales en la fisura sometida a tensiones tangenciales.

Palabras clave: Teoría de corte-fricción, hormigón reforzado con fibras de acero, esfuerzo cortante, ensayos "push-off", deslizamiento a cortante.

\footnotetext{
ABSTRACT

Steel fibre reinforced concrete (SFRC) can be structural in design if it meets the requirements established by structural concrete standards based on residual strengths in bending fracture tests.
} 
However, for shear fracture the use of these residual strengths is still questioned. For this reason, the resistant mechanisms of aggregate interlock and dowel action of the reinforcement, according to the theory of shear-friction in SFRC, continues to be studied. It should be remembered that the fibres improve the behaviour of the material against shear stresses in a crack since they sew the lips of the crack, reducing its opening and increasing the friction of the material. With this background, an experimental campaign on SFRC was designed and developed on push-off tests subjected to direct shear forces to obtain the relative displacements between the sides of the cracks and thus study the validity of the shear-friction theory developed by Walraven. From the comparative study between the experimental results and the analytical shear-friction model, it is concluded that the proposed analytical model is suitable when applied to the behaviour of the SFRC, once the shear fissure has been created, adequately reproducing the normal and tangential displacements in the crack subjected to tangential stresses.

Keywords: Shear-friction theory, Steel fibre reinforced concrete, shear strength, push-off tests, shear slide.

\section{INTRODUCCIÓN}

El refuerzo del hormigón mediante fibras aleatoriamente dispuestas es una técnica ampliamente usada en los últimos 50 años (Conforti et al., 2020, López et al., 2014, Plizzari and Tiberti, 2006). La capacidad resistente de las fibras puede ser considerada en el cálculo estructural del hormigón, si se cumplen ciertas condiciones, debido a que las fibras mejoran la resistencia a tracción y flexión del material (Brandt, 2008). Los requerimientos, para considerar la contribución de las fibras en el cálculo, vienen determinados por diversas normas (American Concrete Institute, 2008, International Federation for Structural Concrete (fib), 2010, Ministerio de Fomento, 2008). Estos requisitos están basados en ensayos de fractura en Modo I (European Committee for Standardization, 2007). Las citadas normativas consideran la capacidad resistente de las fibras en función de las resistencias residuales a tracción por flexión denominadas $f_{R 1}$ y $f_{R 3}$. Sin embargo, para el cálculo de elementos sometidos a esfuerzos diferentes, como a cortante todavía se producen discrepancias a la hora de aceptar como válidas estas resistencias residuales (Conforti et al., 2020, Cuenca et al., 2015, Krassowska et al., 2019, Ortiz-Navas et al., 2020).

Es unánime la opinión de que el refuerzo del hormigón con fibras permite la reducción, e incluso la eliminación, del armado tradicional de cercos (Voo et al., 2010). Pese a ello, todavía existe incertidumbre a la hora de determinar y cuantificar los mecanismos resistentes ante esfuerzos cortantes en el HRFA (Cuenca, 2012). Para poder mejorar en el conocimiento de los mecanismos que se movilizan en un elemento de hormigón fisurado sometido a cortante es necesario identificar las tensiones tangenciales en la zona de hormigón no fisurada, el engranamiento de áridos, el efecto pasador de la armadura longitudinal, el efecto arco y las tensiones residuales de tracción en las fisuras (Taylor, 1974). De tal forma, la teoría más extendida y utilizada para el estudio del esfuerzo cortante sobre el hormigón es la de corte-fricción (Walraven, 1980).

Para poder estudiar la validez de la teoría de corte-fricción cuando ésta se aplica a HRFA fue necesario obtener los desplazamientos relativos entre ambos lados de la sección fisurada. Con tal fin se emplearon hormigones estudiados previamente y que cumplían los requisitos para considerar la contribución de las fibras al comportamiento estructural (Picazo, 2020). Los HRFA empleados estaban reforzados con fracciones volumétricas del $0,64 \%$ y $0,89 \%$. La campaña experimental de obtención de datos se basó en probetas tipo push-off (Echegaray, 2014, Jongvivatsakul et al., 2016) de HRFA sin refuerzo de cercos. Para la obtención de desplazamientos relativos en la fisura se 
empleó un sistema de vídeo-extensometría con el que, además, se obtuvieron los mapas de deformación (Picazo et al., 2018).

La importancia de este estudio radica en la mejora del conocimiento de los mecanismos de engranamiento de áridos y efecto pasador de las fibras en el HRFA en relación a la teoría de cortefricción, ampliamente aceptada para cortante en el hormigón reforzado con barras de acero.

\section{OBTENCIÓN DE DATOS EXPERIMENTALES PARA EL MODELO}

\subsection{Ensayos push-off}

Se emplearon dos hormigones autocompactantes reforzados con 50 y $70 \mathrm{~kg} / \mathrm{m}^{3}(0,64 \%$ y $0,89 \%$ de fracción volumétrica), denominados SCS50 y SCS70. La dosificación del hormigón y las principales propiedades mecánicas, junto con las verificaciones de su capacidad estructural conforme a normas, se encuentran publicadas anteriormente (Picazo, 2020).

Los ensayos realizados fueron del tipo push-off. Las probetas tenían forma de prisma con dos entallas contrapuestas, mostrando la probeta una forma de " $Z$ ". Entre las puntas de las entallas se forma un ligamento plano sobre el que se aplica una carga vertical (Cuenca and Serna, 2010). Las probetas no fueron prefisuradas y la máquina de ensayos empleada tenía una capacidad máxima de carga de $500 \mathrm{kN}$. Los ensayos se realizaron con control de desplazamiento fijando el desplazamiento del actuador a una velocidad constante de $1 \mu \mathrm{m} / \mathrm{s}$. Se dispuso un sistema de vídeoextensometría formado por dos cámaras de $5 \mathrm{MPx}$ encargadas de grabar las secciones de ligamento de las probetas. Este sistema se relacionó con los datos registrados por la máquina de ensayos, lo que permitió obtener de manera sincronizada los datos de tiempo, desplazamiento, carga y deslizamiento a cortante

\subsection{Abertura de fisura y deslizamientos a cortante mediante vídeo-extensometría}

El sistema de vídeo-extensometría permitió obtener las distancias relativas entre diferentes puntos de la superficie de las probetas a lo largo de los ensayos. En la Figura 1(a) se muestra un ejemplo de la colocación de extensómetros virtuales al final de un ensayo. La diferencia relativa de distancias verticales y horizontales entre los puntos refleja la abertura de la fisura y el deslizamiento a cortante.

Con los datos obtenidos en los ensayos se realizaron las curvas de abertura de fisura frente a deslizamiento a cortante. Éstas se muestran en la Figura 1(b) en la que también se muestra la correspondiente a la teoría de corte-fricción (Walraven, 1980) para un hormigón con armadura de refuerzo en la discontinuidad. 

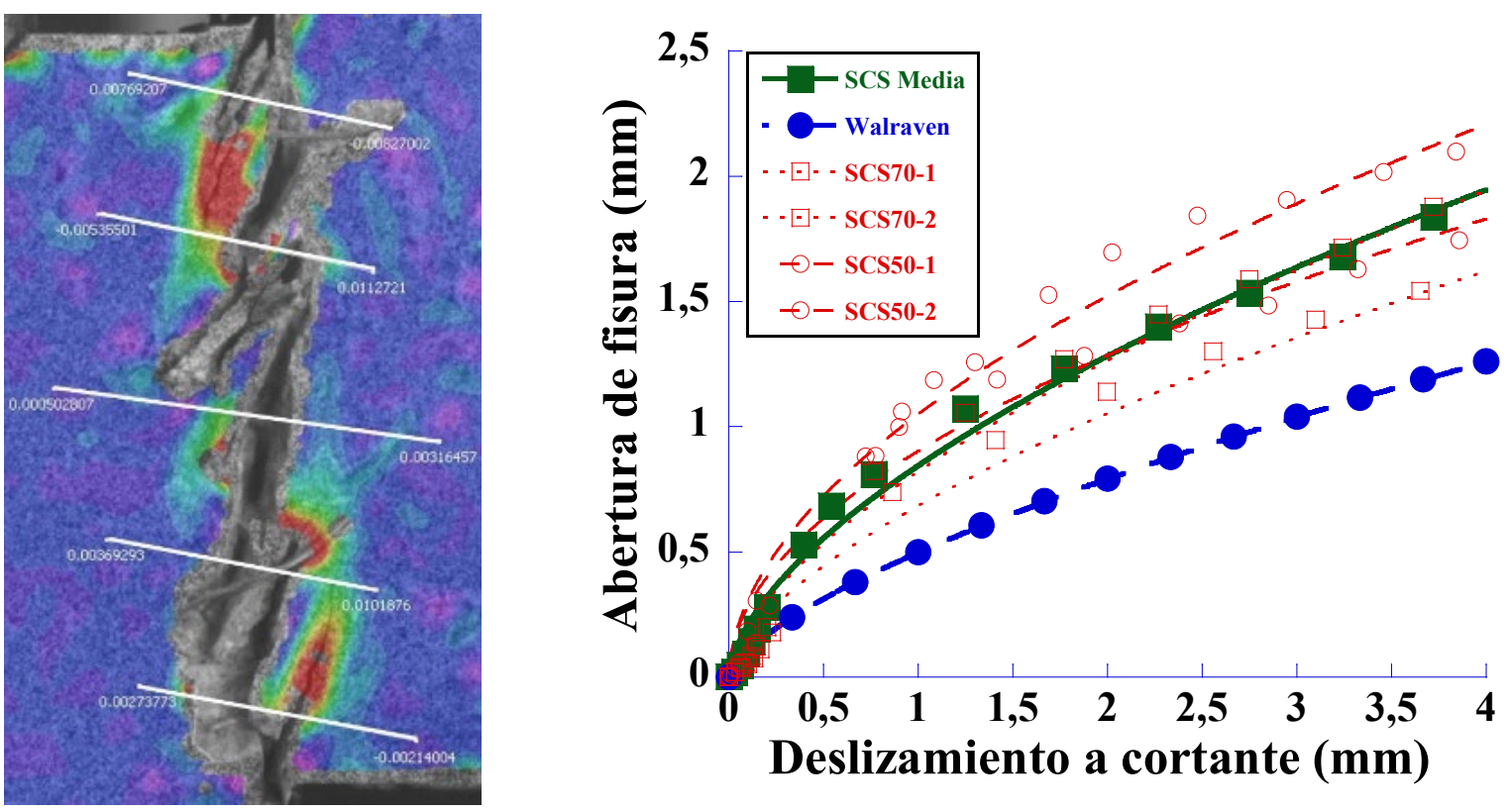

Figura 1. (a) Extensómetros virtuales y (b) gráficas de abertura de fisura frente a deslizamientos a cortante.

Otro factor a considerar a la hora de comparar los resultados analíticos y los experimentales es que, en las probetas, las fibras estaban aleatoriamente dispuestas, en tanto que en el modelo los cercos se disponen de manera favorable para soportar el esfuerzo cortante. Así, en la campaña experimental, solamente las fibras fueron las encargadas de soportar la tensión normal en la fisura.

\section{MODELO DE CORTE-FRICCIÓN}

El modelo de corte-fricción (Walraven, 1980) estudiado en comparación con los resultados experimentales de SFRC sometido a esfuerzo de cortante se puede esquematizar, según aparece en la Figura 2(a): el esfuerzo cortante provoca la generación y desarrollo de fisuras del hormigón produciéndose un deslizamiento relativo entre sus caras. Este desplazamiento se ve impedido por la rugosidad de la intercara y por la acción del refuerzo en la fisura. Si aumenta el esfuerzo cortante se produce el encabalgamiento de una cara sobre la otra, provocando la abertura de la fisura y que las fibras trabajen a tracción evitando dicha abertura y haciendo trabajar al hormigón a compresión en ambas caras. Los principales parámetros a considerar en esta teoría son: el esfuerzo cortante, la resistencia del hormigón, la rugosidad de la intercara y el refuerzo. 

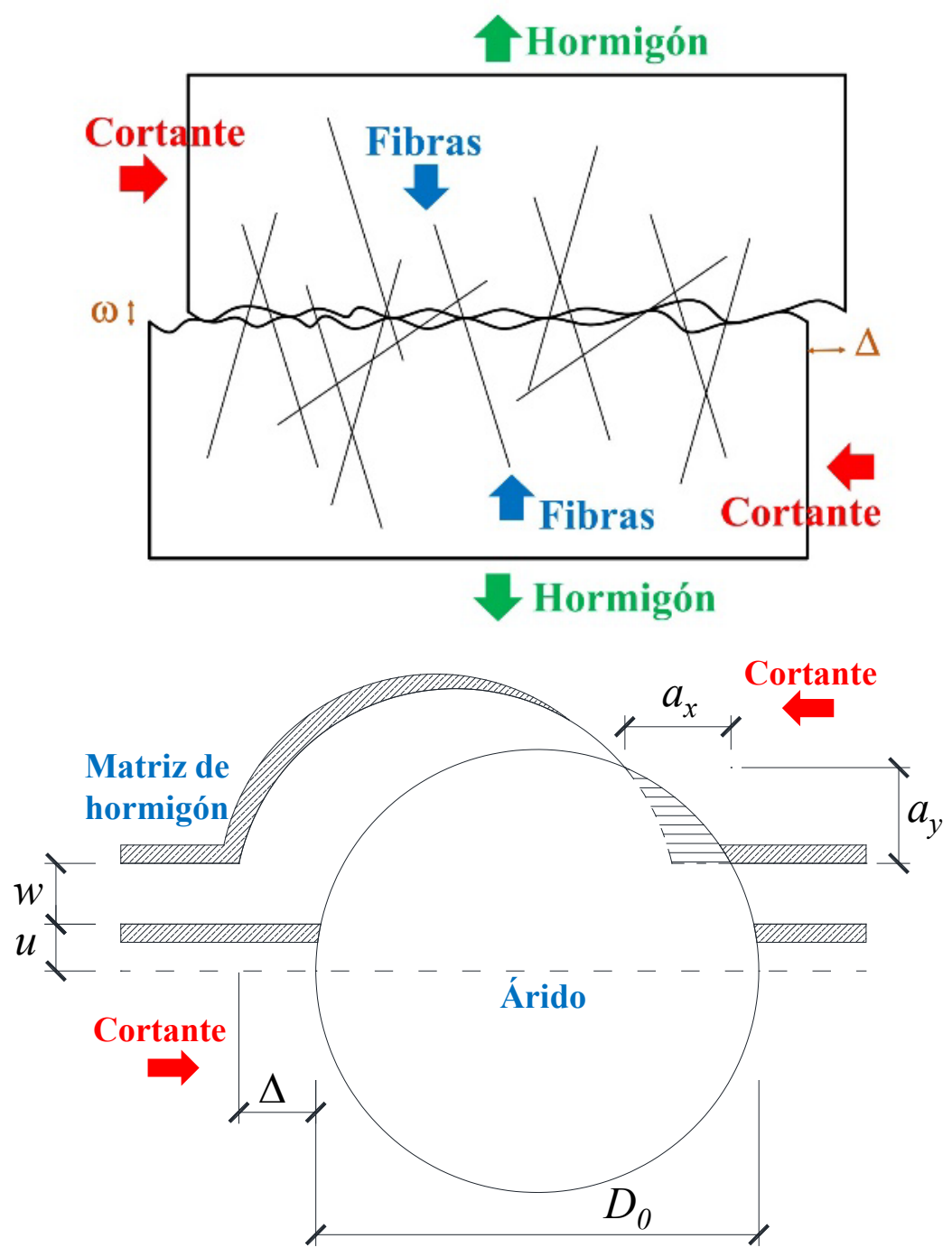

Figura 2. (a) Esquema de los mecanismos resistentes de la teoría corte-fricción y (b) parámetros empleados para el cálculo de la relación entre las proyecciones de las áreas de contacto y los desplazamientos en las caras de la fisura (adaptado de (Walraven, 1980)).

\subsection{Engranamiento de los áridos}

Para poder implementar el modelo de corte-fricción es necesario considerar que la fisura se produce, en hormigones de resistencia normal, en la pasta de cemento, alrededor de los áridos. Igualmente, se realizan las siguientes simplificaciones, la discontinuidad se considera como un plano y los áridos se asumen con geometría esférica. Por último, la matriz del hormigón tiene un comportamiento rígido-plástico. De tal forma, como se puede ver en la Figura 2(b), los áridos de una cara de la fisura provocan un engranamiento con la matriz de la cara contraria por deformación plástica de ésta. Por tanto, existirá contacto árido-matriz que provocará áreas de contacto en los ejes " $x$ " e " $y$ ". De tal forma, las tensiones longitudinales y tangenciales se pueden expresar con las ecuaciones (1) y (2), respectivamente.

$$
\begin{aligned}
& \sigma=\sigma_{p u}\left(A_{x}-\mu \cdot A_{y}\right) \\
& \tau=\sigma_{p u}\left(A_{y}+\mu \cdot A_{x}\right)
\end{aligned}
$$


Siendo: $\sigma_{p u}$ el límite elástico de la matriz, $A_{x}$ y $A_{y}$ el sumatorio de las proyecciones de las áreas de contacto por unidad de superficie áridos-matriz y $\mu$ el coeficiente de rozamiento árido-matriz. Según la Figura 2(b), también existe relación entre los desplazamientos entre las caras de las fisuras ( $w$ es la abertura de fisura y $\Delta$ el deslizamiento por cortante) y las proyecciones de las áreas de contacto por unidad de superficie.

\subsection{Efecto pasador}

El efecto pasador se produce en piezas armadas sometidas a cortante como consecuencia de la resistencia de las barras de refuerzo dispuestas en el plano de la fisura. Este efecto, en el HRFA sin armadura de cercos, tiene que ser soportado por la resistencia a tracción del hormigón y la contribución de las fibras. Dichos mecanismos no tienen un comportamiento frágil, permitiendo la redistribución de tensiones tangenciales en el plano de fisuración. Para considerar el refuerzo de las fibras se empleó la ecuación (3), tomada de la teoría de corte-fricción y adaptada al HRFA.

$$
F_{d f}=\frac{\left(10 \cdot(w+0.2)^{-1} \cdot \Delta^{0.36} \cdot \emptyset_{f}^{1.75} \cdot n^{\underline{o}_{f}} \cdot \theta\right)}{A_{c}}
$$

Donde: $\emptyset_{f}$ el diámetro de una fibra de acero, $n_{f}^{o}$ el número de fibras en la discontinuidad, $\theta$ el coeficiente de orientación de las fibras (Alberti et al., 2016) y $A_{c}$ el área de la sección de hormigón en la discontinuidad.

\subsection{Secciones fisuradas sometidas a esfuerzo cortante con refuerzo de fibras de acero}

En la sección fisurada de HRFA no sólo se producen los mecanismos de transmisión de fuerzas de engranamiento de áridos y efecto pasador. Las fibras son las encargadas de soportar una fuerza normal en la sección que impide que la probeta push-off se rompa en dos mitades. Esta fuerza es función del número de fibras y de su resistencia. Para considerar la resistencia de las fibras se tiene que especificar que se debe considerar el menor valor, entre la resistencia a tracción y la de arrancamiento de la matriz. Esta consideración se demostró experimentalmente durante el conteo de las fibras en la sección de la fisura, ya que todas las fibras de acero se encontraron arrancadas de la matriz, nunca rotas. Para poder implementar en el modelo numérico la tensión generada por la fuerza indicada se utilizó la ecuación (4).

$$
\sigma_{f} \cdot \rho_{f}=\sigma_{c} \Rightarrow \sigma_{f}=\frac{\sigma_{c}}{\rho_{f}}=\frac{\sigma_{p u} \cdot\left(A_{x}-\mu \cdot A_{y}\right)}{\frac{n^{\underline{0}} f \cdot A_{f} \cdot \theta}{A_{c}}}=\frac{A_{c} \cdot \sigma_{p u} \cdot\left(A_{x}-\mu \cdot A_{y}\right)}{n^{\underline{o}} f \cdot A_{f} \cdot \theta}
$$

Donde $\rho_{f}$ es la cuantía de fibras, $\sigma_{c}$ la tensión normal en el hormigón y $A_{f}$ el área de la sección transversal de una fibra.

De tal forma, se produce un equilibrio de fuerzas en la discontinuidad, representado en la Figura 3(a). La fuerza de la máquina de ensayos es la denominada $L_{e}, L_{p}$ es la del efecto pasador, $L_{h}$ la fuerza normal sobre las fibras, $A_{v}$ y $A_{h}$ las fuerzas horizontales y verticales del engranamiento de los áridos y, por último, una fuerza, de valor despreciable, $A_{2}$, correspondiente al engranamiento de los áridos no embebidos en la discontinuidad. 

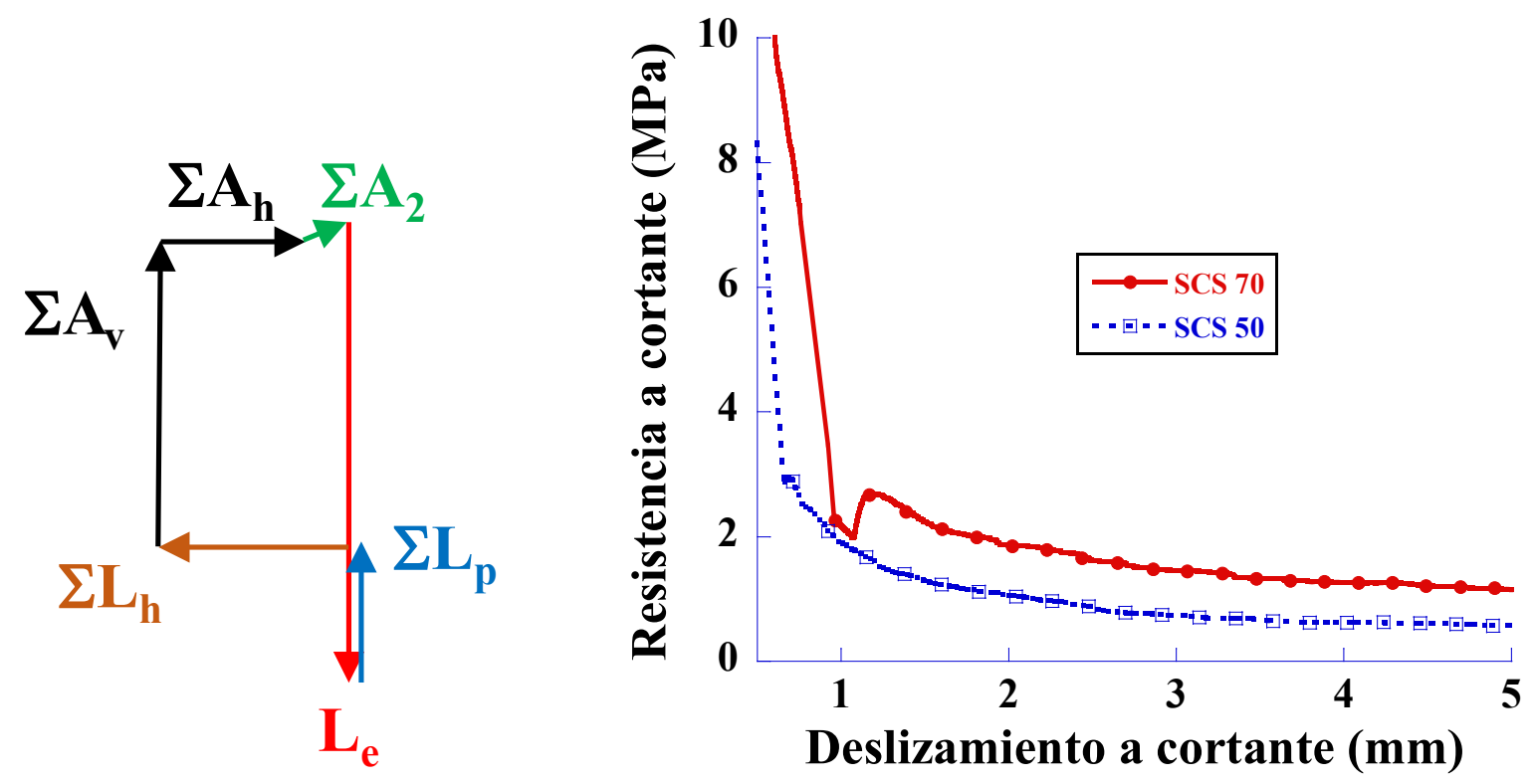

Figura 3. (a) Equilibrio de fuerzas en una discontinuidad reforzada con fibras de acero y (b) detalle, de la zona post-fisuración del HRFA, de la gráfica tensión-deslizamiento a cortante experimental de los HRFA estudiados.

\subsection{Variables a considerar en el modelo numérico de corte-fricción en HRFA}

Las variables consideradas en el modelo numérico de corte-fricción sobre SFRC son: resistencia a compresión del hormigón sin reforzar, diámetro máximo del árido, relación entre el volumen de áridos y de hormigón, coeficiente de rozamiento entre las caras de la discontinuidad, sección de ligamento, abertura crítica del hormigón, diámetro de las fibras de acero, número teórico de fibras, coeficiente de orientación de éstas, resistencia a tracción de las fibras y la tensión de arrancamiento de las fibras.

\section{COMPARATIVA DE RESULTADOS EXPERIMENTALES FRENTE A ANALÍTICOS}

\subsection{Resultados experimentales}

El comportamiento experimental de los dos HRFA Está compuesto por una primera rama lineal de carga hasta el punto de inflexión para producirse a continuación una caída brusca de la misma, aparejada a un deslizamiento a cortante importante. Posteriormente, gracias a la entrada en carga de las fibras, se produjo un descenso poco pronunciado de las resistencias residuales. El comportamiento post-fisuración señalado se muestra en la Figura 3(b). Esta zona del ensayo mostrada en la Figura 3(b) es la que se comparó con el modelo, ya que es cuando las fibras gobiernan el comportamiento post-fisuración del HRFA.

Debido a que en el modelo estudiado existen diversas variables, éstas se tienen que ajustar para cada tipo de hormigón, por lo que se realizó una comparación experimental-numérica para cada hormigón.

\subsection{Comparativa experimental-numérica en el SCS70}

Mediante la introducción de las variables para el SCS70 se pudo realizar la comparativa de los resultados experimentales frente a los obtenidos por el modelo numérico. De tal forma, en la Figura 
4(a) se muestra la gráfica de tensión frente a deslizamiento a cortante para la zona de validez del modelo. Con el fin de centrar la atención en la comparativa buscada se ha eliminado de la gráfica de la Figura 4(a) la zona correspondiente a la rama inicial de carga y parte de la caída brusca posterior al inicio de la fisuración. Se puede ver el comportamiento medio de las probetas ensayadas, junto a su zona de influencia frente al desarrollo numérico analizado. Se puede observar en la Figura 4(a) que a partir de un deslizamiento a cortante, en el entorno de los $2 \mathrm{~mm}$, y hasta un deslizamiento de 4,5 $\mathrm{mm}$ el comportamiento del SFRC según el modelo numérico y experimental resultó muy similar.

De igual forma, se estudió el comportamiento del material respecto a la relación abertura de fisura frente a deslizamiento a cortante. En la Figura 4(b) se puede ver que los resultados del modelo y los hallados experimentalmente experimentales guardan una semejanza evidente. No obstante, hay que señalar que en el caso del modelo se produjo un menor valor de abertura de fisura que en los resultados experimentales, para un mismo valor de desplazamiento. Esta diferencia de comportamiento pudo ser causada por la ausencia de un fisura previa en las probetas ensayadas ya un el modelo se basóen el comportamiento de probetas prefisuradas.

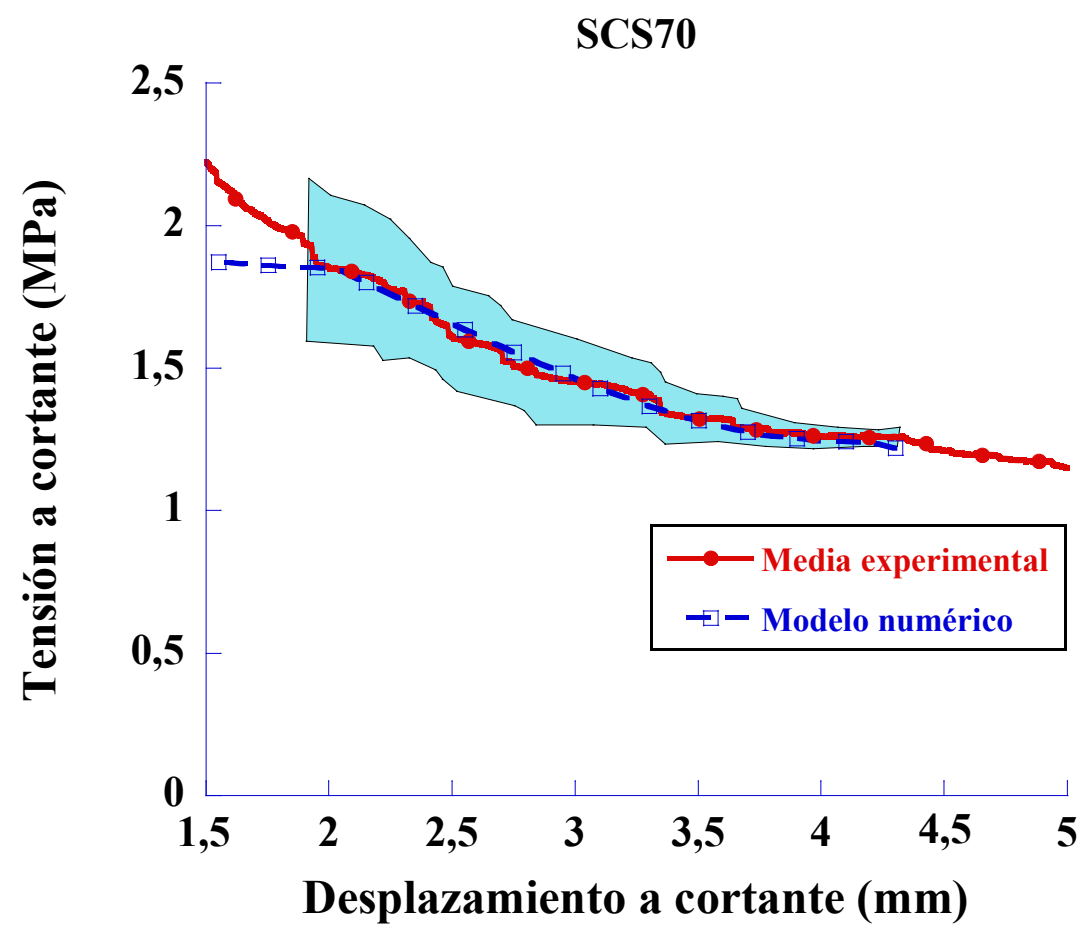




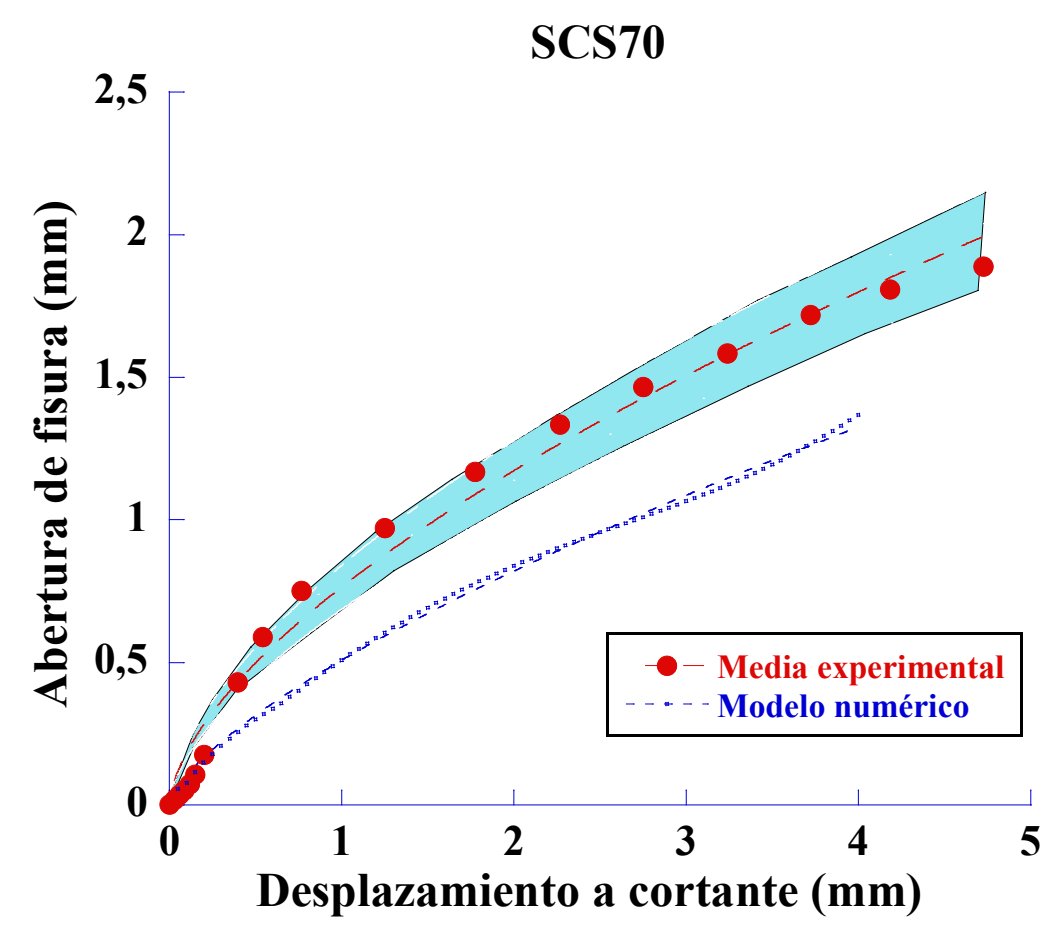

Figura 4. (a) Detalle de la gráfica tensión-desplazamiento a cortante de las probetas SCS70 comparativa experimental-analítica y (b) gráficas abertura de fisura frente a deslizamiento a cortante para el SCS70.

\subsection{Comparativa experimental-numérica en el SCS50}

Análogamente a lo mostrado en la sección anterior, en la Figura 5(a) se observa la comparativa de las gráficas tensión frente a deslizamiento a cortante de los resultados experimentales y la obtenida mediante el modelo analítico. En el caso de los SCS50 la semejanza entre los resultados analíticos y experimentales se muestra a partir de un deslizamiento a cortante menor al del SCS70, en este caso en los 1,5 $\mathrm{mm}$. La similitud de comportamiento de la media de los ensayos frente a la predicción del modelo es importante y se desarrolla hasta un deslizamiento de $4,3 \mathrm{~mm}$. 

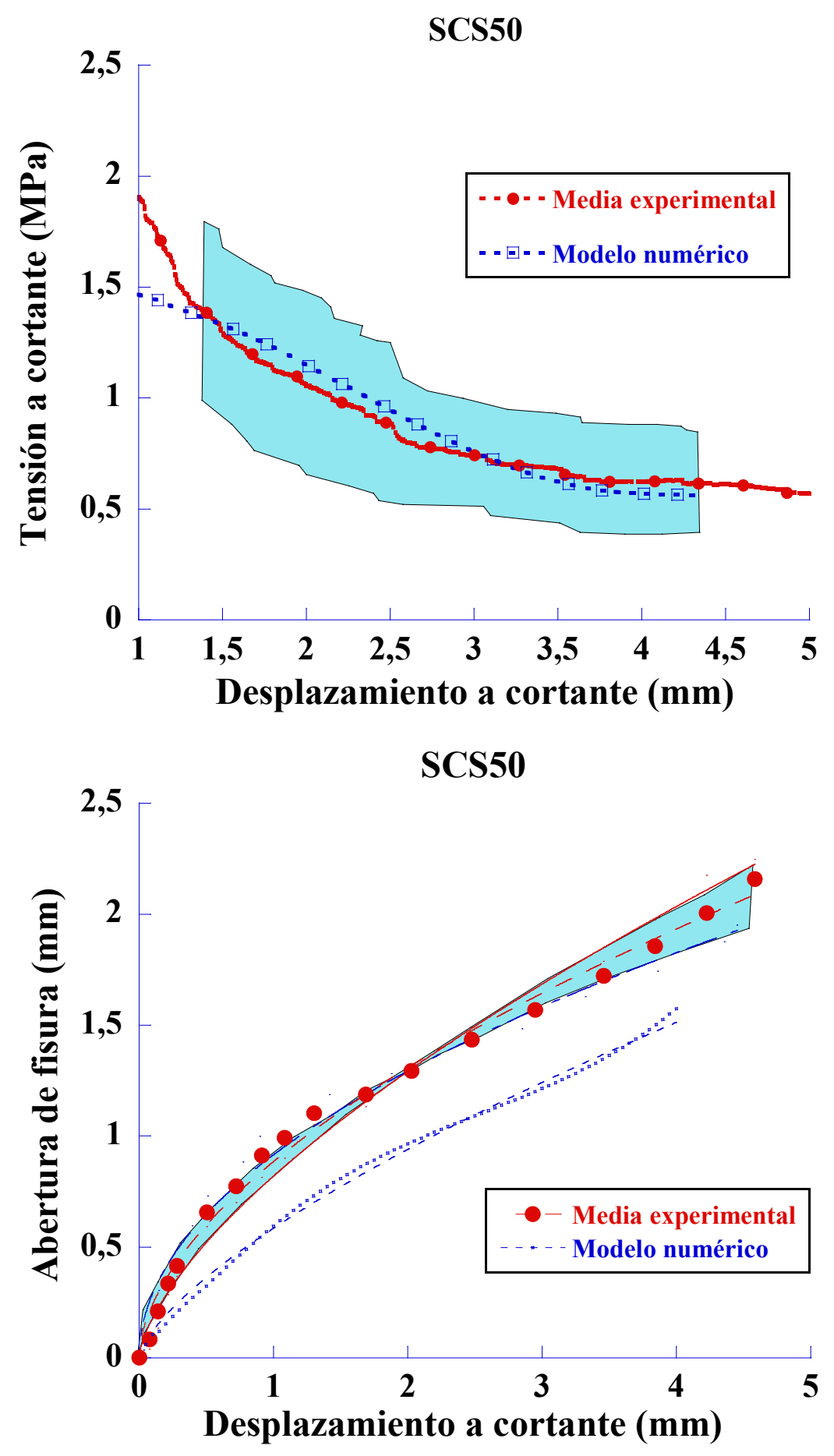

Figura 5. (a) Detalle de la gráfica tensión-desplazamiento a cortante de las probetas SCS50 comparativa experimental-analítica y (b) gráficas abertura de fisura frente a deslizamiento a cortante para el SCS50.

También se desarrolló la compararon los resultados de abertura frente a deslizamiento, tal y como aparece en la Figura 5(b). El comportamiento del SCS50 sigue la misma tendencia que el que mostraba la formulación SCS70 pudiéndose apreciar un un comportamiento similar en el modelo numérico y el experimental. Sin embargo, en el modelo analítico la abertura de fisura es menor en el modelo debido, como se indicó, a que las probetas no fueron prefisuradas. 


\section{CONCLUSIONES}

Se analizó el comportamiento experimental de HRFA sometido a esfuerzos de cortante: éste muestra una rama de carga lineal gobernada por la resistencia de la matriz, tras la cual se produjo un punto de inflexión antes de una rama brusca de descarga asociada a un importante deslizamiento a cortante y abertura de fisura. Tras esta segunda rama se produjo una descarga suave debida a la aportación resistente de las fibras.

Se desarrolló un modelo numérico basado en la teoría de corte-fricción en la rama residual del HRFA sometido a cortante. Para la obtención de los desplazamientos y abertura de fisura se utilizó un sistema de vídeo-extensometría del que se obtuvieron datos válidos para realizar la comparativa experimental-numérica. Los resultados del modelo numérico de corte-fricción se compararon con los experimentales, obteniéndose un buen ajuste.

Una vez conocidos los desplazamientos relativos se pudieron obtener las tensiones en la discontinuidad del HRFA. De tal forma, las resistencias residuales a cortante se podrían integrar a lo largo de las secciones del elemento estructural para poder obtener valores de cálculo en función de su capacidad de carga a cizallamiento.

De la comparativa numérico-experimental en el HRFA sometido a cortante se puede indicar que los ajustes del modelo fueron válidos desde el momento post-fisuración en el que las fibras gobiernan el comportamiento del HRFA.

\section{AGRADECIMIENTOS}

Los autores agradecen el apoyo financiero prestado por el Ministerio de Economía y Competitividad de España a través del Proyecto PID2019-108978RB-C31. También agradecen la contribución de Sika SAU a través de la Cátedra Universidad-Empresa "Cátedra Sika-UPM".

\section{REFERENCIAS}

Alberti MG, Enfedaque A, Gálvez JC, Agrawal V. Fibre distribution and orientation of macrosynthetic polyolefin fibre reinforced concrete elements. Construction and Building Materials. 2016;122:505-17.

American Concrete Institute. 544.3R-08: Guide for Specifying, Proportioning, and Production of Fiber-Reinforced Concrete. Farmington Hills, MI, USA: American Concrete Institute (ACI); 2008.

Brandt AM. Fibre reinforced cement-based (FRC) composites after over 40 years of development in building and civil engineering. Composite structures. 2008;86(1):3-9.

Conforti A, Ortiz-Navas F, Piemonti A, Plizzari GA. Enhancing the shear strength of hollow-core slabs by using polypropylene fibres. Engineering Structures. 2020;207:110172.

Cuenca E, Serna P. Shear behavior of Self-Compacting concrete and Fiber-Reinforced concrete push-off specimens. Design, Production and Placement of Self-Consolidating Concrete: Springer; 2010. p. 429-38.

Cuenca E. On shear behavior of structural elements made of steel fiber reinforced concrete 2012. 
Cuenca E, Echegaray-Oviedo J, Serna P. Influence of concrete matrix and type of fiber on the shear behavior of self-compacting fiber reinforced concrete beams. Composites Part B: Engineering. 2015;75:135-47.

Echegaray J. Upgrading the push-off test to analyze the contribution of steel fiber on shear transfer mechanisms 2014.

European Committee for Standardization C. Test Method for Metallic Fiber Concrete. Measuring the Flexural Tensile Strength (Limit of Proportionality (LOP), Residual); EN14651:2007+A1. 2007.

International Federation for Structural Concrete (fib). The fib Model Code for Concrete Structures 2010. Lausanne, Switzerland: International Federation for Structural Concrete; 2010.

Jongvivatsakul P, Attachaiyawuth A, Pansuk W. A crack-shear slip model of high-strength steel fiber-reinforced concrete based on a push-off test. Construction and Building Materials. 2016;126:924-35.

Krassowska J, Kosior-Kazberuk M, Berkowski P. Shear behavior of two-span fiber reinforced concrete beams. Archives of Civil and Mechanical Engineering. 2019;19(4):1442-57.

López JA, Serna P, Camacho E, Coll H, Navarro-Gregori J. First ultra-high-performance fibrereinforced concrete footbridge in Spain: design and construction. Structural Engineering International. 2014;24(1):101-4.

Ministerio de Fomento MF. Instrucción de hormigón estructural. EHE-082008.

Ortiz-Navas F, Navarro-Gregori J, Leiva G, Serna P. Comparison of macrosynthetic and steel FRC shear-critical beams with similar residual flexure tensile strengths. Structural Engineering and Mechanics. 2020;76(4):491-503.

Picazo Á, Gálvez JC, Alberti MG, Enfedaque A. Assessment of the shear behaviour of polyolefin fibre reinforced concrete and verification by means of digital image correlation. Construction and Building Materials. 2018;181:565-78.

Picazo Á. Estudio experimental y numérico del comportamiento a cortante de hormigones reforzados con fibras de poliolefina y acero: Ph. D. Thesis, Technical University Madrid, Madrid, Spain.; 2020.

Plizzari G, Tiberti G. Steel fibers as reinforcement for precast tunnel segments. Tunnelling and Underground Space Technology. 2006;21(3):438-9.

Taylor HP. The fundamental behavior of reinforced concrete beams in bending and shear. Special Publication. 1974;42:43-78.

Voo YL, Poon WK, Foster SJ. Shear strength of steel fiber-reinforced ultrahigh-performance concrete beams without stirrups. Journal of structural engineering. 2010;136(11):1393-400. 
Walraven JC. Aggregate interlock: a theoretical and experimental analysis. 1980. 